\title{
A simulation model of material particle trajectory by shifting of material by free fall
}

\author{
Vieroslav Molnár ${ }^{1, *}$, and Mikuláš Šveda ${ }^{2}$ \\ ${ }^{1}$ TU Kosice, Faculty of Manufacturing Technologies with seat in Prešov, Bayerova 1, 08001, \\ Slovakia \\ ${ }^{2}$ TU Kosice, Faculty of Mining, Ecology, Process Control, and Geotechnologies, Letná 9, 04201 \\ Kosice, Slovakia
}

\begin{abstract}
The paper is aimed at the calculation of the throwing parabola of material particle and its graphical interpretation by changing input parameters selected according to predefined requirements. The aim is to compile a complex simulation model and comparison of the obtained simulation results on the model with analytical calculation. Simulation experiments on the model confirmed the known dependence of the change of throwing parabola by increased speed and retention of the radius of the staging drum. The simulation model was created in the program MSC Adams/View - a tool for easy construction and visualization of the model and convenient evaluation of the obtained results.
\end{abstract}

\section{Introduction}

Material handling is an important branch of industry [1]. Belt conveyors are in most cases an optimal solution for the continuous transport of mineral raw materials for short and medium distances. The conveyor belt is a key element of conveyor belts. One of the most important areas within the frame of the transport route is the effective flow of materials in the place of shifting. The places of shifting of the transported materials are widely used in the industry, including mining, processing of raw materials, chemical processing, thermal power plants and many other areas that process solid particle [2]. Hou and Meng [3] dealt with dynamic properties and determination of the working performance of the conveyor belt.

One of the possibilities of examination of contact forces and dynamic resistance of conveyors is to create simulation models. Nuttall et al. [4] presented a simplified approach to modeling of rolling-resistance contact on the surface of the roller and rubber belt. He examined parameters of the model with viscoelastic properties and more complex Maxwell models in terms of rolling friction and traction. There are many methods available for analysis of particle flow by conveyor including continuous analytical methods, DEM and experimental analysis. The most well-known applied method, examining the interaction of material particle at the point of shifting, is the discrete element method (DEM - Discrete Element Method). Simulation experiments based on this method are presented by the most authors in order to optimize and design elements of shifting. Research of interaction caused

* Corresponding author: vieroslav.molnar@tuke.sk 
by the contact of particles flow during impacts on the wall of shifting is presented by Gröger and Katterfeld [5], Kessler and Prenner [6], Grima and Wypych [7], [8] and Dewicki [9]. David and Kruses [10] described the input parameters obtained by simulation in the application DEM (for example static or dynamic load of material, material density, particle adhesion and cohesion, restitution coefficient, viscosity). Di Renzo and Di Maio [11] investigated the course of forces, speed and displacement during collisions of material particles. Chandramohan and Powell [12] verified by DEM simulation a standard model of viscous damping and compared experimental and numerical results. Bierwisch et al. [13] investigated rapid granular flow using numerical simulations in DEM. Jensen et al. [14] presented a simulation model in DEM that models particles of general shape by a combination of several smaller particles with simpler shape than a circle into clusters that act as one larger particle. Zhang and Xia [15] applied a modeling approach based on optimization to improve the efficiency of conveyor belts operation.

The aim of the paper is to compile a complex simulation model of the material particle trajectory by material shifting with freefall and comparison of the obtained simulation results on the model with the analytical calculation.

\section{The mining equipment specification}

Shifting is the place where the conveyed material is transferred from on belt conveyor to the other (Fig. 1). The task of shifting is to concentrate the material flow and direct the conveyed material to the middle of the next belt with the lowest falling height. In these places, the material usually falls by free fall, chute or feeder. The concentrating of the material to the point of impact is realized by constructional suitable selection of the shape and setting of the precipitating plate and the placement of the hopper. The type of conveyed material has a great impact on the lifetime of the belt, especially by shifting of material from the belt to the belt. Abrasive and bulk materials have another way of the structural solution than sticky material. For this reason, the structural solution must have some changes for this type of conveyed material.

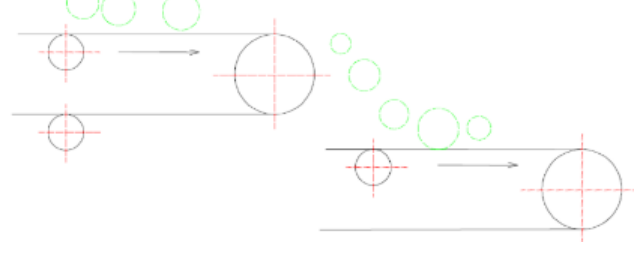

Fig. 1. Shifting of material by free fall without a feeder. Source: Author

In order to prevent cracks and damage of the conveyor belt, it used spring-loaded idler rollers, or rubberized rollers under shifting formed damping layer.

Another way is to compress rollers under shifting (Fig. 2a) or using of impact shatter place with impact bars in the place of impact (Fig. 2b).

Compression of rollers under shifting is the most commonly used type of design, in which the only flexible element is a conveyor belt, that damps and catches all kinetic energy of the conveyed material and at the same time, it keeps the direction of transport. There is a frequent break due to the point contact of the belt - roller, inelasticity of the construction of rollers clamping, deflection of the conveyor belt from the direction of running due to the unequal effect of the conveyed material and difficult backward return of the belt to the original direction.

The construction of the impact place should provide sufficient stiffness, elasticity, ability 
to guide the conveyor belt in the transport direction, absorb the kinetic energy of the flatling material, provide the surface contact of the material with the conveyor belt by reducing of the frictional resistance and option of easier sealing of the environment. The impact bars are positioned on the support construction. This construction is assembled at the point of top part of conveyor belt route so that the impact of the material on the conveyor belt damps the impact of the material and thus reduced the impact energy of the material.

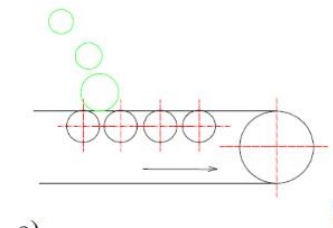

a)

b)

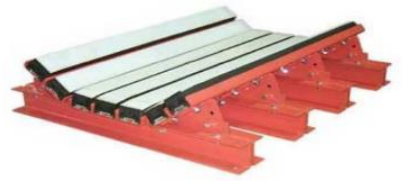

Fig. 2. Reduction of conveyor belt damage a) compression of rollers in the place of impact b) construction of impact place with impact bars in the place of impact. Source: Author

The cause of conveyor belt wear can be [16]:

- $\quad$ excessive overloading of the conveyor belt,

- filling and impact of the material on the conveyor belt by uncontrolled manner,

- deflection of material against lateral support of the conveyor belt,

- reverse angle and direction of material filling as the direction of the transferee belt conveyor.

Sealing systems (Fig. 3) are used for this reason, and their main advantage is:

- they do not use strong pressure on the belt,

- the primary seal is on the inside of the conveyor belt,

- they keep the material in the hopper,

- secondary seal captures fine material,

- the finest particles are suppressed on the belt by sealing slats,

- minimal setting.

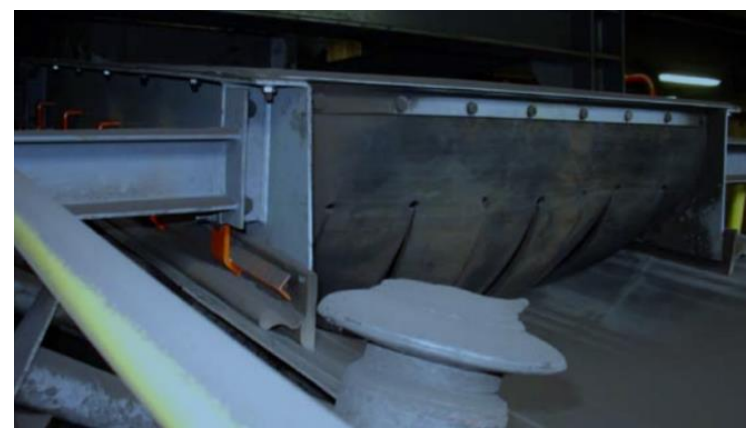

Fig. 3. A sample of sealing system of the conveyor belt. Source: Author

\section{Creation of a simulation model in the environment of ADAMS/View}

\subsection{Environment of ADAMS/View}

ADAMS (Automatic Dynamic Analysis of Mechanical Systems) is a computational system for modeling and simulation of mechanical systems consisting of solid and flexible 
bodies bound to one another through various types of kinematic bonds. It is one of the most widely used systems for static, kinematic and dynamic analysis of designed mechanical models. It also allows to optimize and verify their mathematical models [17]. The module ADAMS/View allows to solve tasks of kinematics and dynamic therefore different virtual prototypes of mechanical systems can be created. These are formed by joining of individual bodies (bulk or flexible) by means of links to the whole unit. For the most accurate assembly of the system model, springs, bumpers, contacts, friction, forces, moments, etc. are used. Measurers "measure" is used for obtaining input analyses.

Basic terms used in the environment ADAMS/View:

- model - a mechanical system composed of bodies, bonds, forces, markers, etc.,

- bodies - bulk or flexible, connected by links. They are defined by weight, geometry, position, etc.

- $\quad$ Links - they define the movement of bodies (translational, rotating, spherical, etc.).

For the creation of a simulation model, it was used a tool for easy construction and visualization and comfortable evaluation of the obtained results by ADAMS/View. By the creation of a new model, it is possible to choose the type of coordinate system, unit system, the direction of action and value of gravity acceleration.

\subsection{Input parameters of the simulation model}

The same input parameters by Table 1 were used for analytical calculation and simulation model.

Table 1. Input parameter of the shifting model

\begin{tabular}{|c|c|c|c|c|}
\hline $\mathbf{v}_{\mathbf{0}}\left[\mathbf{m} \cdot \mathbf{s}^{-1} \mathbf{]}\right.$ & $\mathbf{R}[\mathbf{m}]$ & $\mathbf{m}[\mathbf{k g}]$ & $\mathbf{h}[\mathbf{m}]$ & $\mathbf{g}\left[\mathbf{m} \cdot \mathbf{s}^{-2} \mathbf{]}\right.$ \\
\hline 2 & 0,6 & 0,5 & 3 & 9,81 \\
\hline
\end{tabular}

where:

$\mathrm{v}_{\mathrm{o}}$ - the speed of the conveyor, value by [1], and this is the normalized speed of belt conveyor $\left[\mathrm{m} . \mathrm{s}^{-1}\right]$

$\mathrm{R}$ - radius of staging drum, the value is by the diameter of the staging drum with a rubber-textile belt $[\mathrm{m}]$

$\mathrm{m}$ - the weight of the material particle, selected value $[\mathrm{kg}]$

$\mathrm{h}$ - height of material shifting, selected value [m]

$\mathrm{g}$ - gravity acceleration $\left[\mathrm{m} \cdot \mathrm{s}^{-2}\right]$.

Before model creation in ADAMS/View, it is needed to know the mathematical model of the bound mechanical system that we want to model and analyze. Different forces can act on the bodies, different passive effects can be introduced in kinematic bonds, deformable bonds can be among bodies (springs, buffers, etc.). Bodies may have a certain movement. By comparison of dynamic forces with analytical calculation, it was considered with material shifting by the effect of one particle. Table 2 presents components of the model of shifting.

For our project, bodies were created, the parameters are presented in Table 2. The horizontal bases (bodies with the shape of a cylinder) present a conveyor belt with the staging drum (body with the shape of the roller). 
Table 2. Components of the simulation model of shifting

\begin{tabular}{|c|c|c|c|c|c|}
\hline & length [m] & width [m] & height [m] & radius [m] & weight [kg] \\
\hline $\begin{array}{c}\text { Horizontal } \\
\text { base }\end{array}$ & 2 & 0,2 & 0,2 & - & - \\
\hline $\begin{array}{c}\text { Horizontal } \\
\text { base }\end{array}$ & 5 & 0,2 & 0,2 & - & - \\
\hline $\begin{array}{c}\text { Material } \\
\text { particle }\end{array}$ & - & - & - & 0,01 & 0,5 \\
\hline
\end{tabular}

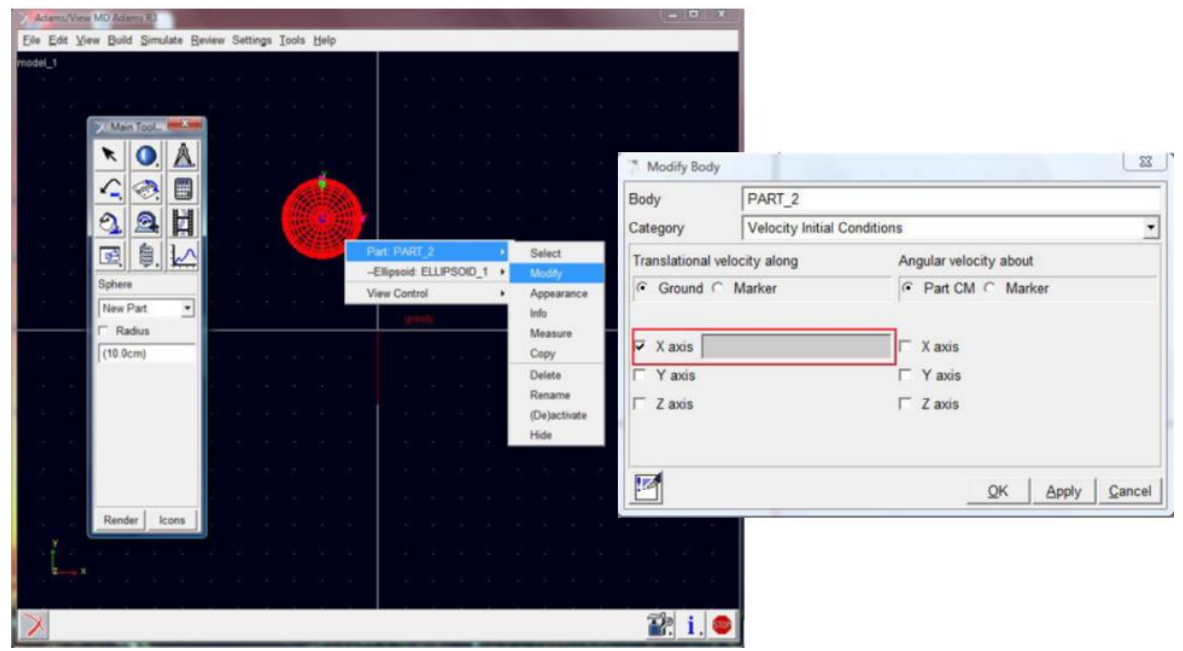

Fig. 4. Creation of particle and definition of parameters of movement. Source: Author

In order to determine the time of material leaving from the horizontal base (conveyor) as well as the impact on the second horizontal base, it is necessary to define the contacts in the model of shifting (Fig. 5):

- moving of material over a horizontal base,

- moving along the staging drum,

- impact and moving along the second horizontal base.

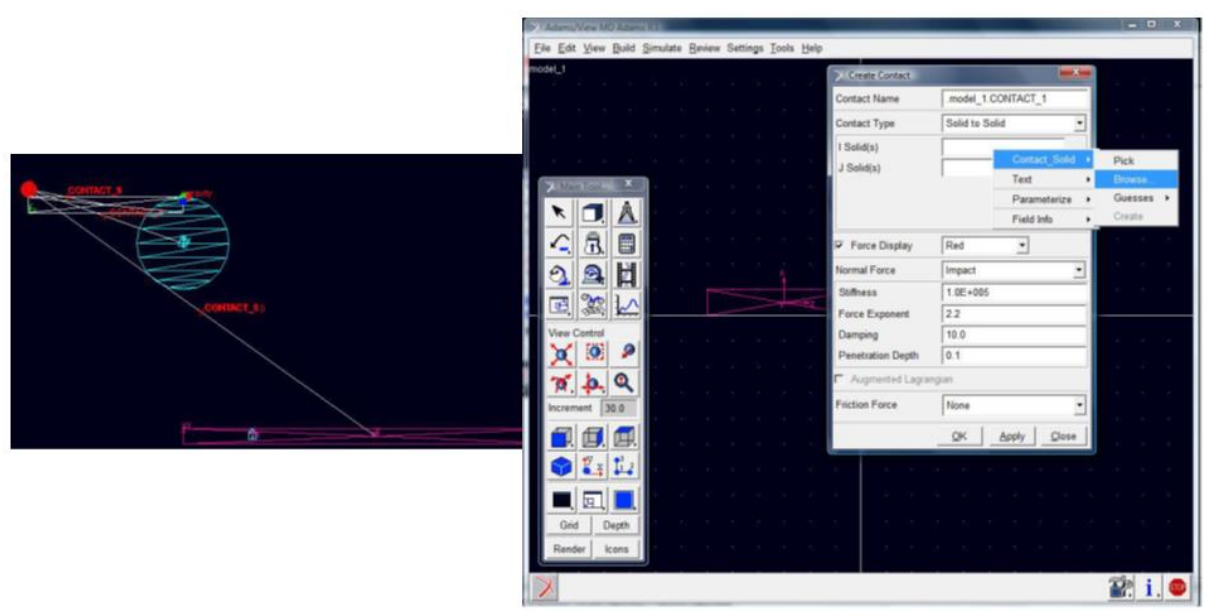

Fig. 5. Definition of particle contacts with components of shifting (horizontal bases). Source: Author 


\subsection{Simulation experiments}

Simulation experiments are conditioned on the functionality of the designed simulation model. An important part of the analysis is the selection of the monitored quantities. Measurers "measures" are used in the system ADAMS/View. The measurer is created by editing of a body whose parameters we want to measure. Outputs from the measurer are a shift, speed, acceleration, and reactions. The measurer can monitor the course of the selected quantity directly during the simulation in a separate window. Fig. 6 presents the measurer of the particle motion in the direction of the axis $y$.

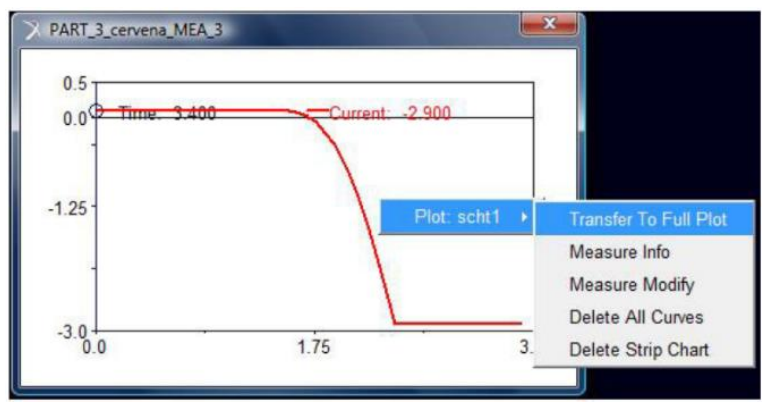

Fig. 6. The trajectory of the particle in the axis y. Source: Author

The solution neglected adhesion of the material to the conveyor belt, sliding of particles along the circumference of staging drum as well as the air resistance. The model particle is a steel pellet.

\section{Results and Discussion}

On the horizontal base (conveyor), the particle has the speed $\mathrm{v}_{0}$ and at the same time, this speed is a speed of the belt. The blue circle with the radius $\mathrm{R}=0,6 \mathrm{~m}$ presents the staging drum of the conveyor belt. Overhanding and transferee conveyors have the same direction of material movement. Fig. 7 presents the created model of shifting and trajectory of throwing parabola with the input data from the Table 1 and Table 2.

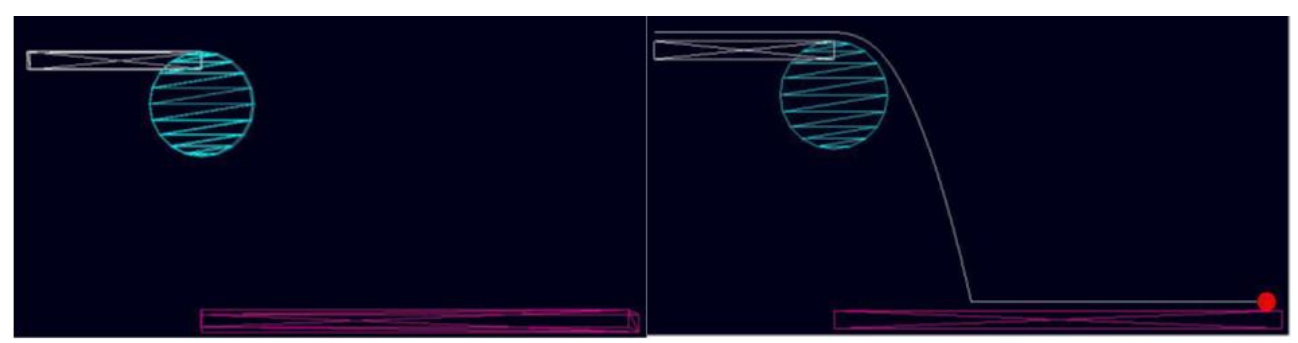

Fig. 7. Model of shifting and trajectory of the particle for $\mathrm{v}_{0}=2 \mathrm{~m} \cdot \mathrm{s}^{-1}$ in ADAMS/View. Source: Author

Results of the throwing parabola were evaluated in the module "Post Processor" (Fig. 8). 


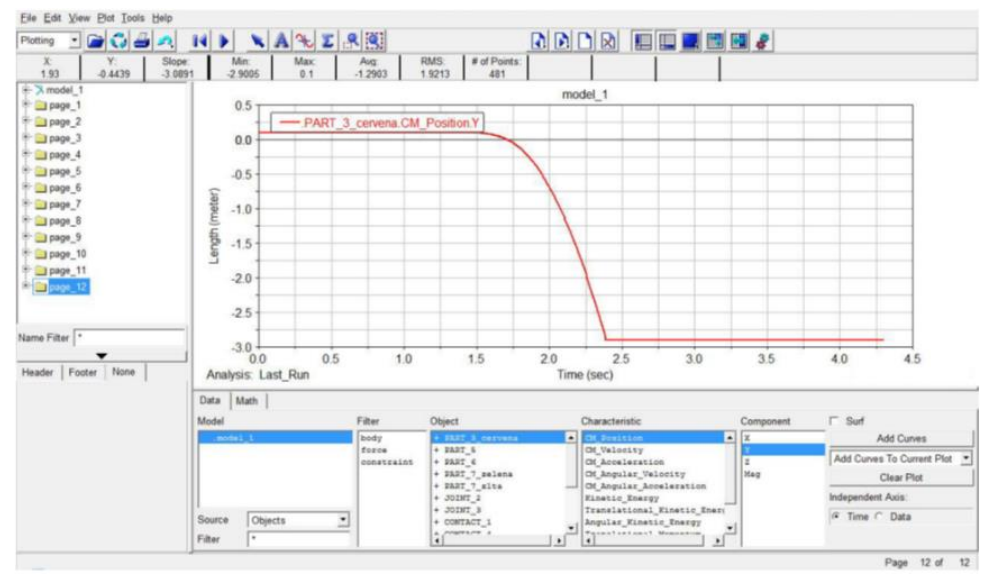

Fig. 8. Results of throwing parable in the module "Post Processor". Source: Author

The time of particle falls from one base to the other $\mathrm{t}=0,64 \mathrm{~s}$ which is identical with analytical calculation. The particle separates from the staging drum in the time $t=1,75 \mathrm{~s}$ (Fig. 9).

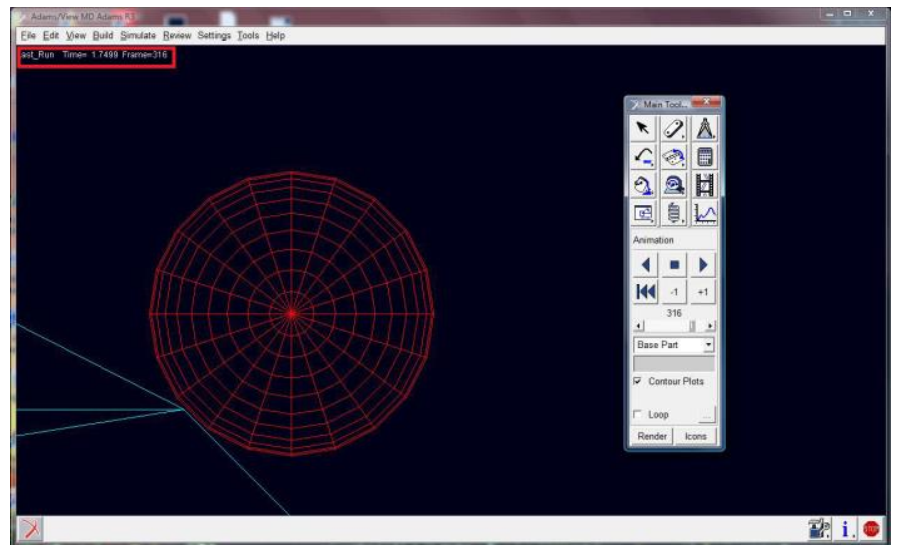

Fig. 9. Determination of the time of material separation from staging drum in the time $t=1,75$ with ADAMS/View. Source: Author

Fig. 10a presents an increase of the trajectory of particle separation from the staging drum at a time, where the yellow line presents a particle with the speed $\mathrm{v}_{0}=4 \mathrm{~m} \cdot \mathrm{s}^{-1}$, weight $\mathrm{m}=0,5 \mathrm{~kg}$ which separates from the staging drum with the angle $\alpha=47^{\circ}$ before the modeled particle (red line). The particle with the speed $\mathrm{v}_{0}=4 \mathrm{~m} \cdot \mathrm{s}^{-1}$ touches the second base (conveyor belt) as the first. The time of particle separation from the staging drum and impact on the second base is greater than the time of the modeled particle (red line). The particle with the speed $\mathrm{v}_{0}=2,5 \mathrm{~m} \cdot \mathrm{s}^{-1}$ (green line) has the same input parameters of the yellow and red line and behaves similarly. By increasing of the speed $\mathrm{v}_{0}$, with the same weight of the particle and the angle of through $\alpha$, by simulation experiments, it was demonstrated that the trajectory and time of particle impact to the second horizontal base increase with the constant height of shifting. Fig. $10 \mathrm{~b}$ presents the particle with the speed $\mathrm{v}_{0}=4 \mathrm{~m} \cdot \mathrm{s}^{-1}$ (yellow course) and its contact with the base as the first. This particle overcomes the longest time from particle separation to the contact with the second base. The particle of green color impacts later. The modeled particle (red course) contacts the base as the last one and confirms the validity of 
the analytical calculation of the time of impact on the second conveyor. The trajectory of the throwing parable for three different initial speeds $\mathrm{v}_{0}$ is presented by Fig. 11.

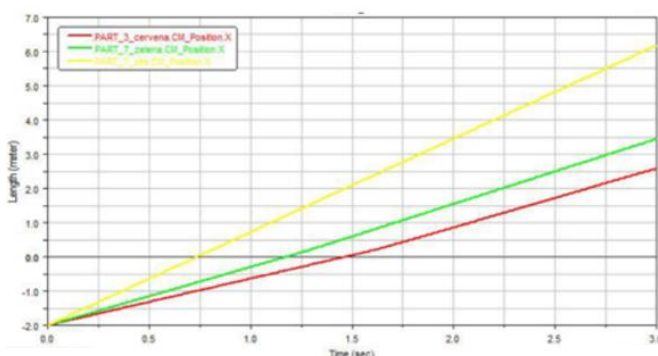

a)

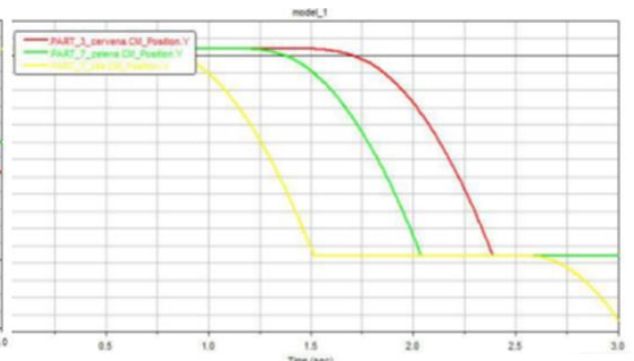

b)

Fig. 10. Results a) increase of trajectory of particle separation from the staging drum, b) changes of throwing parable in the module "Post Processor" for three different initial speeds vo. Source: Author

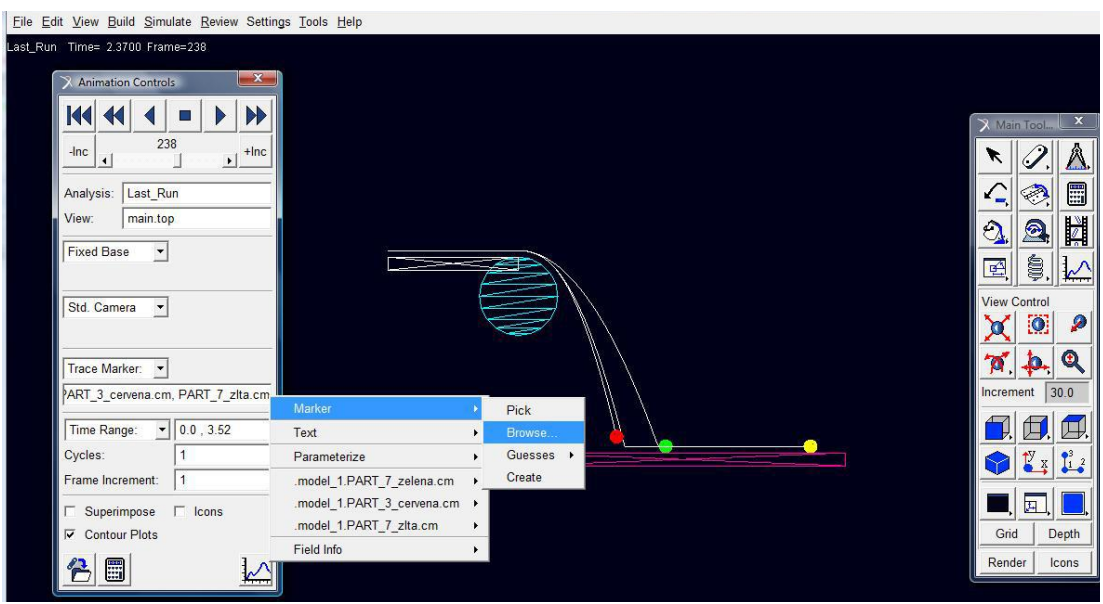

Fig. 11. Trajectories of throwing parable for three different initial speeds $v_{0}$ in ADAMS/View. Source: Author

\section{Conclusion}

A belt conveyor has an irreplaceable place in many technological processes of mining and processing industry. In the course of material conveying among belt conveyors, it is possible to monitor its shifting, or dumping and material are separated from the staging drum with a certain angle. The constructional solution of shifting is an important aspect of the energy efficiency of the conveyor equipment with the aim to minimize the occurrence of critical situation and failures of the conveyor belt. Another issue is the direction and orientation of material impact which has direct effect on the energy efficiency of the conveyor. The size of the angle of a material particle through depends on the speed of belt conveyor, the diameter of the staging drum at the end of the belt conveyor and the type of conveyed material. The paper describes the creation and verification of the universal simulation model in which it is possible to monitor the progress of the changes of predefined parameters in the module "Post Processor". The experiment showed the correspondence of the coordinates of throwing parable and the time of material impact on the conveyor belt with analytical calculation. 
The present paper is a part of research grant projects VEGA 1/0063/16, VEGA 1/0403/18, KEGA 018TUKE-4/2016.

\section{References}

1. S. Zhang, X. Xia, Appl. Energy 88 (2011)

2. A. G. Huque, S. T. McLean, Bulk Solids Handl. 22 (2002)

3. Y. Hou, Q. Meng, J. China Univ. Min. Technol. 18 (2008)

4. A. J. G. Nuttall, G. Lodewijks, A. J. Klein Breteler, Wear 260, (9-10) (2006

5. T. Gröger, A. Katterfeld, Bulk Solids Handl. 27 (2007)

6. F. Kessler, M. Prenner, FME Trans. 37 (2009)

7. A. P. Grima, P. W. Wypych, Granul. Matter 13, (2010)

8. A. P. Grima, P. W. Wypych, Eng. Comput. 28 (2011)

9. G. Dewicki, Bulk Solids Handl. 23 (2003)

10. Conveyor Belt Transfer Chute Modeling and Other Applications using The Discrete Element Method in the Material Handling Industry [online], Available from: http://www.ckit.co.za/secure/conveyor/papers/troughed/conveyor/conveyor.htm, (2016)

11. A. Di Renzo, F. P. Di Maio, Chem. Eng. Sci. 59 (2004)

12. R. Chandramohan, M. S. Powell, Miner. Eng. 18 (2005)

13. C. Bierwisch, T. Kraft, H. Riedel, M. Moseler, J. Mech. Phys. Solids 57 (2009)

14. R. P. Jensen, P. J. Bosscher, M. E. Plesha, T. B. Edil, Int. J. Numer. Anal. Methods Geomech. 23, (1999)

15. S. Zhang, X. Xia, Appl. Energy 88 (2011)

16. C. Benjamin, P. Donecker, S. Huques, The Transfer Chute Design Manual for Conveyor Belt Systems (National Library of Australia Catalouging, 2010)

17. MSC Software [online] Available from: http://www.mscsoftware.com/product/adams 